\title{
Coronary angiography enhancement for visualization
}

\author{
Shengxian Tu • Gerhard Koning • \\ Joan C. Tuinenburg • Wouter Jukema • \\ Su Zhang · Yazhu Chen · Johan H. C. Reiber
}

Received: 26 March 2009/ Accepted: 10 July 2009/Published online: 26 July 2009

(C) The Author(s) 2009. This article is published with open access at Springerlink.com

\begin{abstract}
High quality visualization on X-ray angiograms is of great significance both for the diagnosis of vessel abnormalities and for coronary interventions. Algorithms for improving the visualization of detailed vascular structures without significantly increasing image noise are currently demanded in the market. A new algorithm called stick-guided lateral inhibition (SGLI) is presented for increasing the visibility of coronary vascular structures. A validation study was set up to compare the SGLI algorithm with the conventional unsharp masking (UM) algorithm on 20 still frames of coronary angiographic images. Ten experienced QCA analysts and nine cardiologists from various centers participated in the validation. Sample scoring value (SSV) and observer agreement value $(\mathrm{OAV})$ were defined to evaluate the validation result, in
\end{abstract}

S. Tu $(\bowtie) \cdot$ G. Koning · J. C. Tuinenburg ·

J. H. C. Reiber

Department of Radiology, Leiden University Medical Center, Leiden, The Netherlands

e-mail: S.T.Tu@lumc.nl

S. Tu · G. Koning · J. C. Tuinenburg · J. H. C. Reiber Medis Medical Imaging Systems b.v., Leiden, The Netherlands

W. Jukema

Department of Cardiology, Leiden University Medical Center, Leiden, The Netherlands

S. Zhang - Y. Chen

Department of Biomedical Engineering, Shanghai Jiao Tong University, Shanghai, China terms of enhancing performance and observer agreement, respectively. The mean of SSV was concluded to be $77.1 \pm 11.9 \%$, indicating that the SGLI algorithm performed significantly better than the UM algorithm $(P$-value $<0.001)$. The mean of the OAV was concluded to be $70.3 \%$, indicating that the average agreement with respect to a senior cardiologist was $70.3 \%$. In conclusion, this validation study clearly demonstrates the superiority of the SGLI algorithm in the visualization of coronary arteries from X-ray angiograms.

Keywords Contrast enhancement .

Lateral inhibition $\cdot$ Noise $\cdot$ Stick

\section{Introduction}

Coronary angiography is a minimally invasive procedure that requires the administration of a contrast agent via a catheter into the coronary arteries to visualize the inside by lumen [1]. It is performed during both diagnostic and interventional procedures. During the passage of the contrast agent through the coronary arteries, images are acquired with an angiographic X-ray system at 12.5 or more frames/s. Because of the low pass characteristics of X-ray systems, the sharpness of the visualized coronary arteries is limited (images are blurred), which become 
especially visible when zooming in on interesting parts of the image for observing its detailed structures. In certain cases, e.g., branching vessels or complex lesions, high quality visualization of certain anatomical information is of great significance for the diagnosis. Therefore, post image enhancement, a process by which the image is manipulated to achieve a better perception or interpretability of the information in the image, could assist cardiologists in appreciating the finer details of the coronary anatomy.

There are some factors in the area of angiographic image enhancement which have been widely accepted by cardiologists:

- The image enhancement is used for visualization purposes only, and not for quantitative analysis. Possible effects of image enhancement on the accuracy and precision of quantitative coronary arteriography (QCA) have been investigated [2]. A definite effect was clearly demonstrated, especially for QCA on vessels with smaller diameters $(<1.2 \mathrm{~mm})$. Therefore, it is advisable that enhancement be used for visualization purposes only, and that the original images are kept for archiving and quantitative analysis purposes.

- Detailed image structures should not be lost during the enhancing procedure. Achieving nice appearance and contrast at the sacrifice of losing some detailed information is not acceptable. Image enhancement is expected to improve the visibility of vascular structures with diagnostic value. Therefore, image details should not "disappear" after enhancement.

- The original dimensions of vascular structures should be preserved in the enhanced image. Any change of the dimensions, e.g., overestimation or underestimation of arterial diameters, could introduce a twisted interpretation, resulting in an inappropriate clinical decision.

The literature on enhancing X-ray coronary angiographic images for visualization purposes is very limited. Although a number of algorithms have been proposed for angiographic image enhancement, the purpose of most algorithms is to improve subsequent segmentation rather than visualization. These algorithms can hardly be adopted in clinical practice for improving visualization quality because of the aforementioned factors. Algorithms based on specific noise models, e.g., quantum noise model [3], might also fail to work in practice since image noise, i.e., the undesirable appearance of mottled or grainy spots which do not reflect true tissue property, is the hybrid of various sources of noise with different characteristics. Attempting to increase the contrast of vascular structures by suppressing or removing background structures, e.g., the piecewise normalization [4], the rolling algorithm [5], are also of limited effect, since part of image noise with intensity value within the range of foreground structures, e.g., vessels, will be enhanced as well. The step of removing the background might at the same time remove some detailed information in low contrast angiographic images, which is very undesirable.

To the best of the authors' knowledge, all angiographic acquisition systems available on the market use a certain technique to enhance the acquired images in real time, i.e., during the actual acquisition procedure. Most of these enhancement techniques are based on the so-called unsharp masking technique, and allow the operators to customize the degree of enhancement by using multiple gain levels (typically five). The unprocessed image is first blurred and subtracted from the original image, creating an edge image that only contains the higher spatial frequency components of the original image. This edge image is further multiplied by a certain gain level and added to the original image, resulting in an edge enhanced image [2]. Although image edges are visually enhanced, the result is less optimal since image noise with high spatial frequency will also be enhanced, which might introduce undesirable appearance or influence the perception of the image details.

We have been very interested in developing a technique for enhancing image details without the aforementioned negative effects, e.g., the increase of noise level. A new nonlinear enhancement model, which is called stick-guided lateral inhibition (SGLI), is presented in this paper for improving the visualization of vascular structures, in particular for coronary arteries. The proposed model simulates the enhancing mechanisms integrated in the eyes of human beings and of many animals. By integrating asymmetric sticks as a main tool to approximate vessel edges information for guiding the inhibition process, it has the ability to accentuate the intensity gradients of interesting vessel edges, while suppressing the increase of noise. In this paper the performance of SGLI is compared with the unsharp 
masking (UM) algorithm implemented on the Philips Digital Cardiac Imaging (DCI) System (Philips Medical Systems, Best, the Netherlands) [2]. In the following sections, the methodological background will be presented, as well as the clinical materials, the set up of the validation study, followed by the presentation of the results, the discussions and the conclusions.

\section{Methods}

Original lateral inhibition model

The earliest phases of the visualization process in the human being begin in the retina. Signals resulting from light falling on the photoreceptors are first processed by various interactions among retinal neurons, of which the lateral inhibition network is an instance. The retinal neurons receive excitatory input from overlying photoreceptors as well as inhibitory inputs from adjacent illuminated photoreceptors to shape the signals and pass them on by optic nerve to higher visual centers. It is the laterally spread inhibition feature that gives "lateral inhibition" networks their name [6]. Figure 1 is a schematic diagram illustrating how lateral inhibition functions in the retina. Green bars represent photoreceptors, which function as signal generators according to the amount of light falling on them. Red circles represent output neurons, which integrate excitatory input signals from overlying photoreceptors (indicated by solid vertical lines) and inhibitory input signals from adjacent photoreceptors (indicated by dash diagonal lines). The output will be passed on to higher visual centers. This phenomenon was first observed and investigated in the eye of the Limulus [7-10]. It has been shown that the interactions among the receptor units (ommatidia) in the eye of the Limulus are predominantly inhibitory and obey simple linear relationships [9].

One important function of the inhibitory interactions in the retina is contrast enhancement. On the image edge where the illumination changes, the inhibition from receptor units at the brightly lit side outweighs the inhibition from receptor units at the dimly lit side, resulting in different decreases of signal at two sides. In addition, receptor units are deployed spatially and the strength of their interaction depends on their separation: the inhibition generally decreases

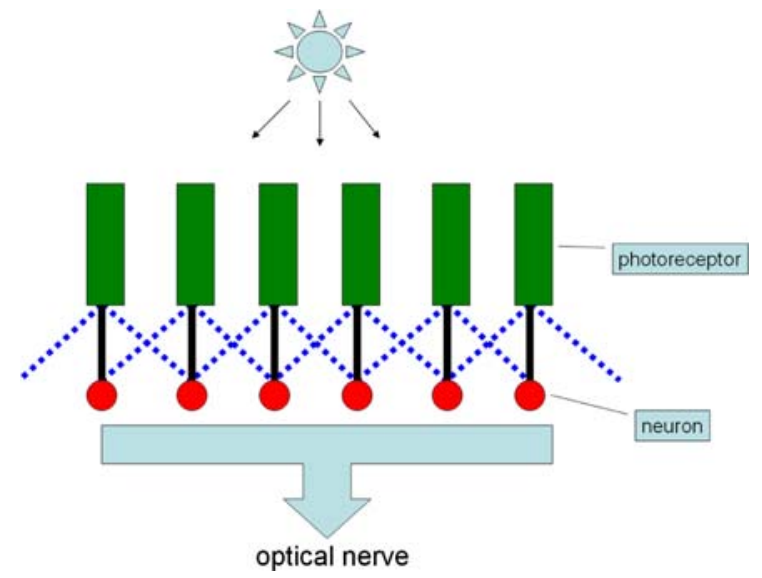

Fig. 1 Lateral inhibition network (only the inhibition from the direct neighbors is indicated for illustration purposes)

as the distance of interacting units increases. Hence, adjacent receptor units exert a stronger inhibition on each other than distant units, the discrepancy of activities among adjacent receptors, especially for those units around the edge, increases. Such mechanism has been widely adopted in enhancing image edge contrast. An example is given by Fig. 2. A and B represent brightly and dimly lit areas, respectively. $\mathrm{E}$ is the image edge. Clearly, the contrast of the image edge increases after inhibition.

Despite of its simplicity, the original lateral inhibition model has limited capacity in enhancing low contrast images due to its sensitivity to image noise. The model needs some "guidance" in order to work effectively on low contrast X-ray images.

\section{Stick-guided lateral inhibition}

The most challenging part of the guiding procedure is to distinguish vascular structures from image noise. Once an acceptable estimation of vessel edges is achieved, the contrast of vascular structures can be improved without increasing image noise in homogenous regions, e.g., background and lumen. In one of our papers [11], we used asymmetric sticks as a tool to perform the task of estimating image edges in a noisy background. Each stick is a digital line with certain direction. Since vessel edges can be decomposed into multiple digital lines, certain combinations of sticks could be used to approximate edges information. 
Fig. 2 Image contrast enhancement by lateral inhibition model

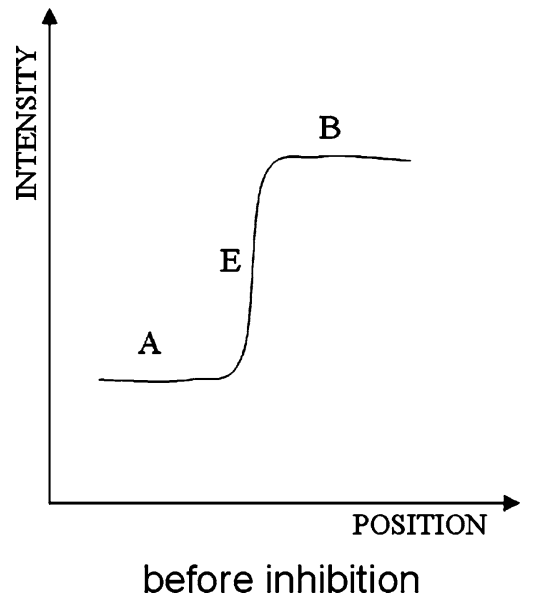

The stick technique for image processing was first proposed by Czerwinski et al. [12, 13] and further extended by Xiao et al. [14] by introducing asymmetric sticks. Compared with symmetric sticks, asymmetric sticks can better approximate image edges, since image edges, especially for the curved parts of edges, are generally asymmetric. Figure 3 shows an asymmetric stick filtering kernel with length 4 . Given the stick length as $L$, a stick filtering kernel contains $8 L-L$ different asymmetric sticks with the same starting point, the center of each squared panel.

By increasing angular resolution, the stick filtering kernel is able to detect digital edges with different directions. Statistical features along these sticks are used in the SGLI model to approximate vessel edges information. Based on the edges information, the degree of inhibition will change adaptively for each image point. The proposed SGLI model optimizes the enhancement of vessel edges by avoiding enhancing image noise.

Figure 4 shows the enhancement results by different lateral inhibition models. Figure $4 \mathrm{a}$ is the original angiographic image (only part of the image is shown). The image is a bit blurred. The lesion near the bifurcation is not clearly visible. Figure $4 \mathrm{~b}$ shows the enhanced result by the original lateral inhibition model. Although the visibility of vascular structures
Fig. 3 Asymmetric stick filtering kernel with length four

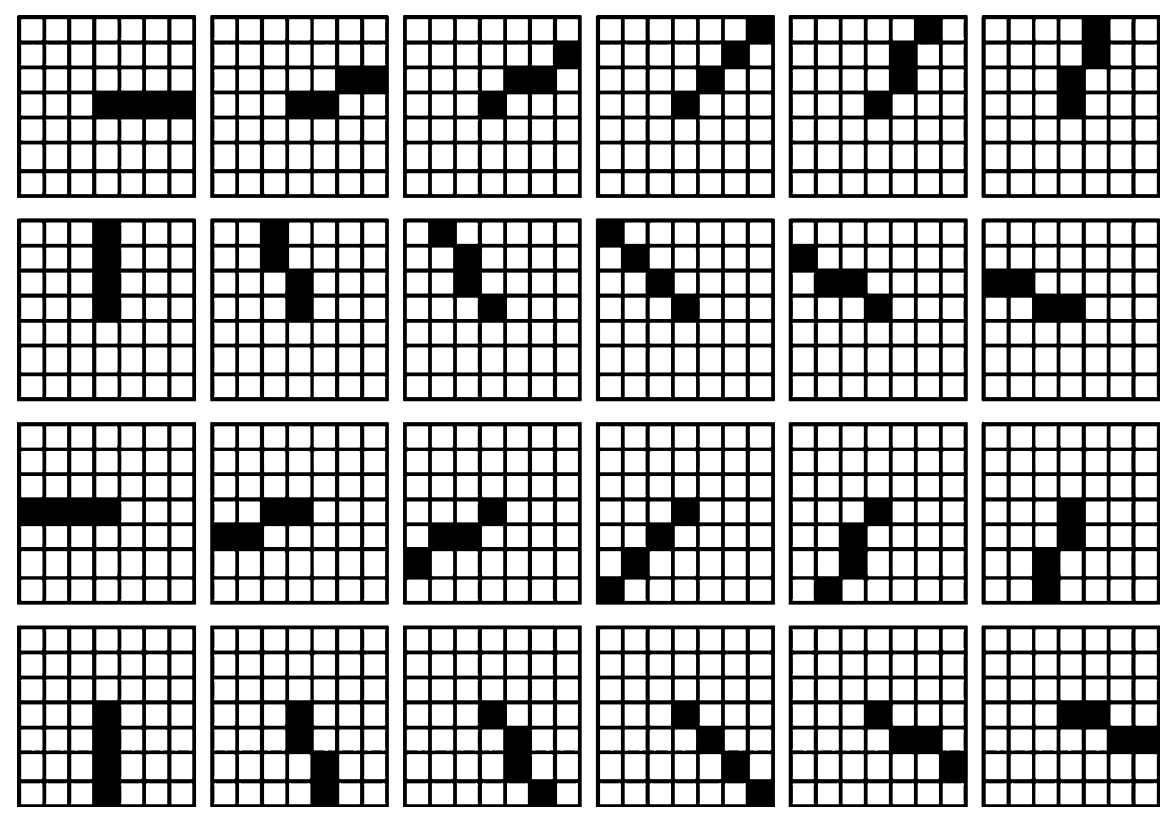


Fig. 4 Angiographic image enhancement by lateral inhibition models: $\mathbf{a}$ is the original image; $\mathbf{b}$ is the result of enhancement by the original lateral inhibition model; $\mathbf{c}$ is the result of enhancement by the improved lateral inhibition model without guidance; $\mathbf{d}$ is the result of enhancement by stickguided lateral inhibition model
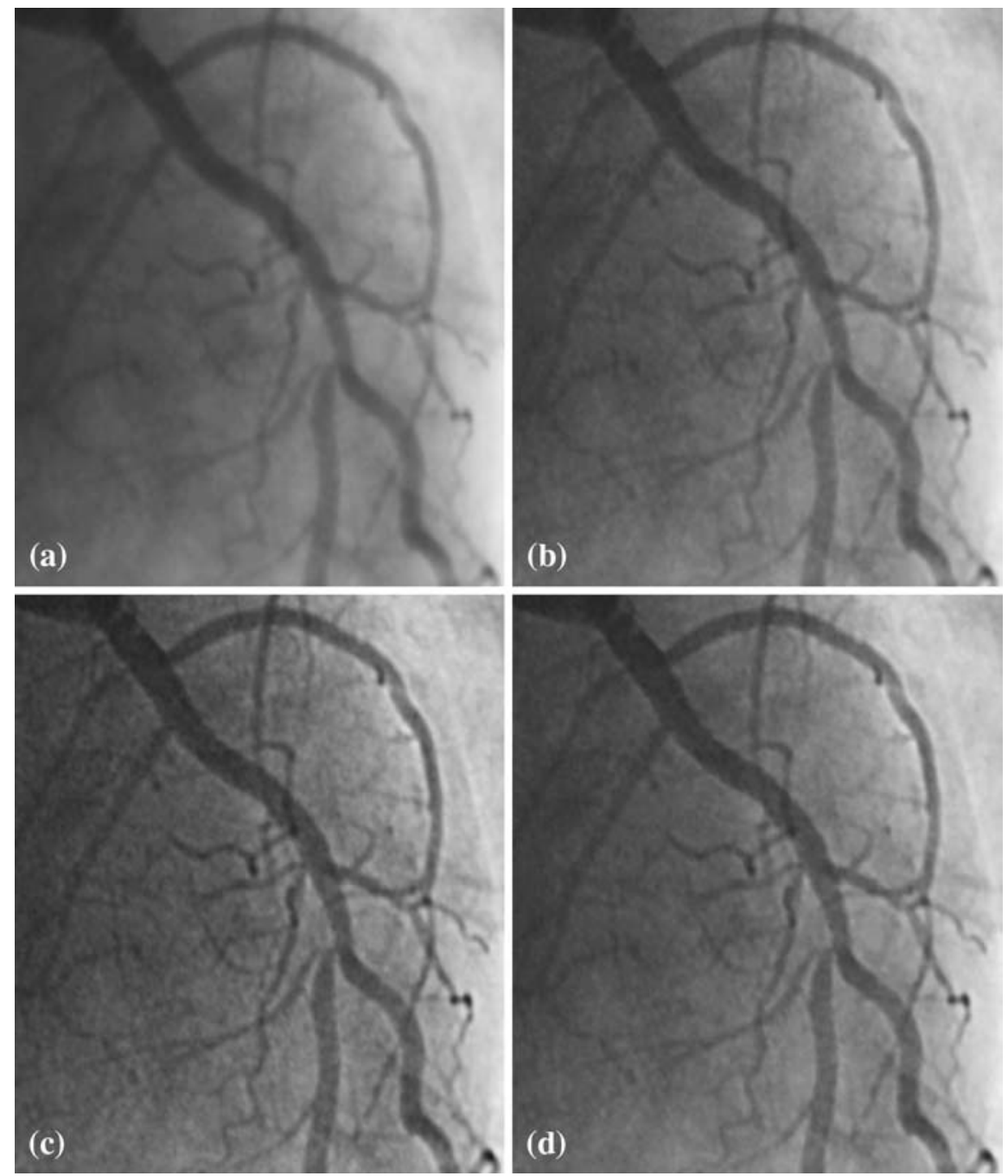

increases, the improvement is moderate. To enhance the detailed information further, a guided inhibition term (GIT) was introduced as a general framework to improve the performance of the lateral inhibition model [11]. The GIT used the edge properties of the image point with respect to its neighbors to adjust the degree of enhancement for that specific image point. The properties could be simply assigned as fixed values (without guidance) or obtained by statistical estimation using the stick filtering kernel (with sticks guidance). Figure $4 c$ and $d$ show the results of enhancement by implementing GIT without guidance and with sticks guidance, respectively. Clearly, vessel edges in both enhanced images look sharper than those in the previous versions. The lesion near the bifurcation is better visualized and appreciated. However, the enhancement algorithm without guidance apparently increases the noise level, resulting in a lot of undesirable grainy spots. On the contrary, SGLI significantly enhances the visualization of the vascular structures, while keeping the noise at a low level. Therefore, the quality of visualization improves.

\section{Validation}

At the Leiden University Medical Center, routinely acquired coronary angiographic images with different noise levels from 15 patients were selected from the clinical databases; images were acquired by the Philips Cardiac Integris systems with $512 \times 512$ image resolution; critical information related to patients had been made anonymous before the 
Fig. 5 An example of the grouped image pair for comparing the SGLI and the UM

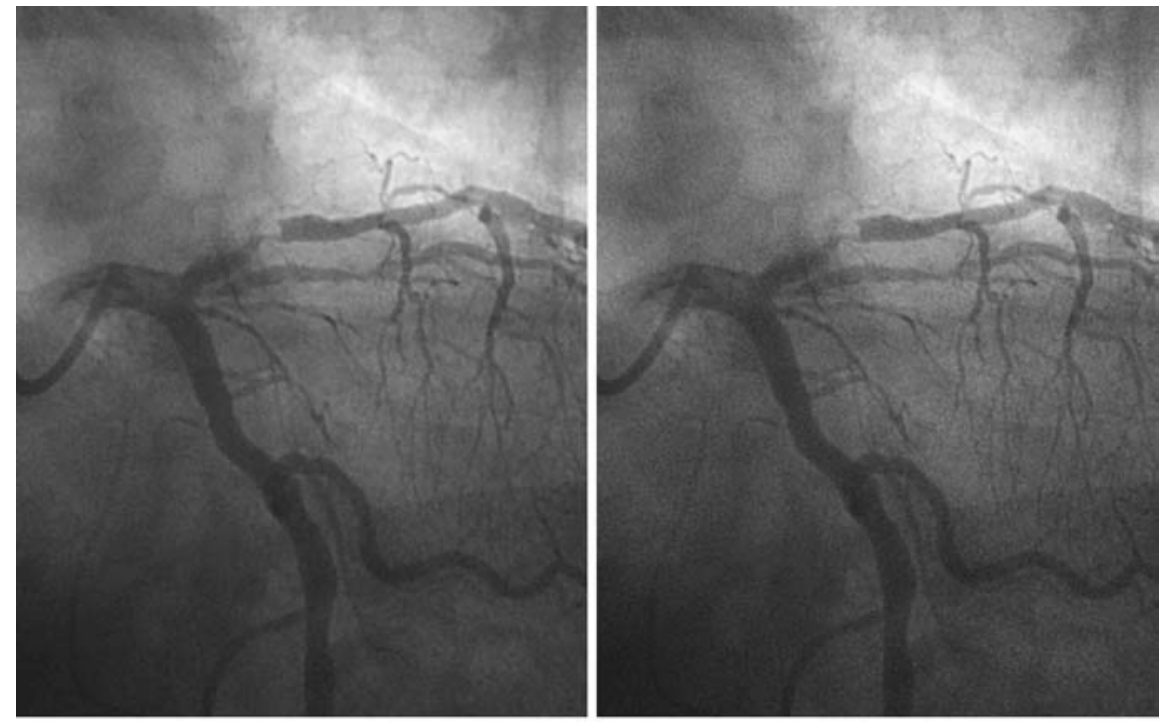

Slice 1 validation. Twenty still image frames at different phases of cardiac circle with clinically relevant information were selected from the data set by experts for the validation.

The validation study was to compare the performance of SGLI and UM algorithms on the selected 20 image frames. Nineteen participants including ten QCA analysts and nine cardiologists from five hospitals in the Netherlands, Japan, Brazil, China, and America participated in the validation. For each image frame, SGLI and UM were applied with the same level of enhancement, which was set by the experts for optimally visualizing the images. The enhanced versions by SGLI and by UM with the same region and zooming factor were grouped into one image pair and incorporated into a PowerPoint slice. Each slice shows the SGLI enhanced version and the UM enhanced version with the same level of enhancement. Figure 5 shows an example of the prepared PowerPoint slices. The left-right position of these two enhanced images was randomly set, i.e., the left image could be the SGLI enhanced version or the UM enhanced version. Therefore, the participants were blind to the enhancement algorithm undertaken by each individual image.

In the scoring procedure, the participants were asked to indicate which image (the left image or the right image) in each slice is the better enhanced image. Given the fact that there is still no gold standard for evaluating the quality of visualization, we chose the following three features to be considered for a good enhancement result:

1. Enhance the detailed information which could increase the real diagnostic value.

2. Enhance the sharpness of vessel edges which could improve the contrast of the vascular structures.

3. Keep the noise as low as possible so that interesting information is easier to be appreciated and image looks more pleasant.

It is our belief that the ability to visualize more detailed information should be the first priority for an enhancement algorithm, followed by the reduction of effort in interpreting the interesting information and pleasant appearance of the image content. Therefore, the following steps were set up to approach the scoring procedure:

Step 1: Look thoroughly at two enhanced images in the same slice. Choose the image with clearer detailed information as the better image.

Step 2: If there is no difference in the detailed information between two enhanced images, then the image with sharper vessel edges is the better image.

Step 3: If there is still no difference on the edges sharpness between two enhanced images, then the image with less noise should be the better image. 
Fig. 6 Comparisons of

SGLI and UM on one

angiographic image: $\mathbf{a}$ is original angiographic

image; $\mathbf{b}-\mathbf{d}$ are the images enhanced by UM with gain level 1, 3, and 5; $\mathbf{e}-\mathbf{g}$ are the images enhanced by SGLI with gain level 1,3 , and 5
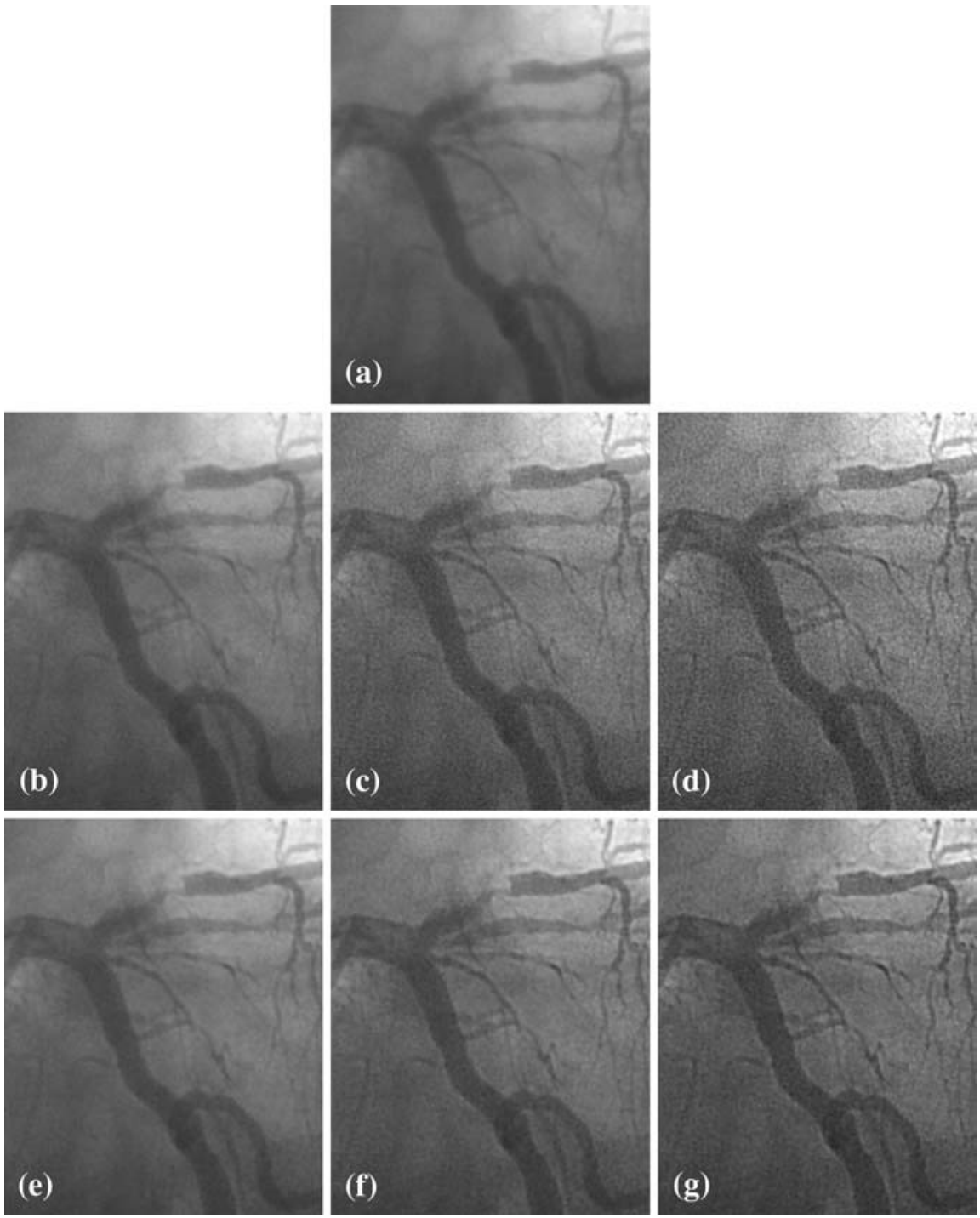

\section{Statistics}

After the scoring procedure, results were mapped into two categories:

Category A: The SGLI enhanced version is better than the UM enhanced version.

Category B: The UM enhanced version is better than the SGLI enhanced version.

Based on the mapping result, two parameters, the sample scoring value (SSV) and the observer agreement value (OAV), in terms of enhancing performance and observer agreement, respectively, are defined to evaluate the scoring result.
1. The SSV is defined by the percentage of observers (participants) belonging to Category $\mathrm{A}$ and is calculated for each sample (slice). The mean of the SSV was computed and considered to be an index to the superiority of the SGLI enhancement algorithm with respect to the UM algorithm. Fifty percent represents equal performance between these two algorithms. SSV above $50 \%$ indicates that the SGLI algorithm is better and SSV below 50\% indicates that the $\mathrm{UM}$ algorithm is better. One-sample $t$ test was performed to investigate whether the mean of SSV is significant different from the $50 \%$ value. 
2. The OAV is defined by the percentage of agreement between one senior cardiologist and the other observers and is calculated for each observer except for the senior cardiologist. The senior cardiologist with an extensive experience in interventional cardiology was thus defined to be the gold standard against whom the others were compared. The mean of OAV represents the average agreement with respect to the senior cardiologist.

All statistical analyses were carried out by using statistical software (SPSS, version 16.0; SPSS Inc; Chicago, IL, USA).

\section{Results}

Visual interpretation

The proposed SGLI algorithm was compared with the UM algorithm available as the enhancement algorithm on the Philips Digital Cardiac Imaging System [2]. We set five gain levels of enhancement for the SGLI algorithm to make it comparable to the UM algorithm. An example of comparison between these two algorithms is given by Fig. 6. Figure 6a is the original angiographic image. Figure $6 \mathrm{~b}-\mathrm{d}$ show the images enhanced by the UM algorithm with the lowest, median, and highest gain level, respectively. With the increasing amount of enhancement, the edges of vascular structures look sharper and sharper. However, image noise also increases significantly. A lot of grainy spots appear in both lumen and background on the enhanced images. Figure $6 \mathrm{e}-\mathrm{g}$ shows the images enhanced by the SGLI algorithm with the lowest, median, and highest level, respectively. With the increasing amount of enhancement, vascular structures also become clearer and clearer while image noise has relatively slight increase. Therefore, the enhancement result is more appreciated. At lower levels of enhancement, the difference between these two algorithms is moderate, although the vessel edges in the SGLI enhanced image still look a bit sharper. At higher levels of enhancement, the difference becomes quite obvious.

Quantitative results

The value of SSV for each sample is given by Fig. 7 . The mean of SSV is $77.1 \%$, with a standard deviation of $11.9 \%$. There is significant difference between the mean of SSV and the $50 \%$ value $(P$-value $<0.001)$, indicating that the observers show significant preference on the SGLI enhanced images.

Figure 8 shows the OAV for each observer. The mean of the OAV is $70.3 \%$, indicating that in average the observers agree with the senior cardiologist on
Fig. 7 The sample scoring value for each sample

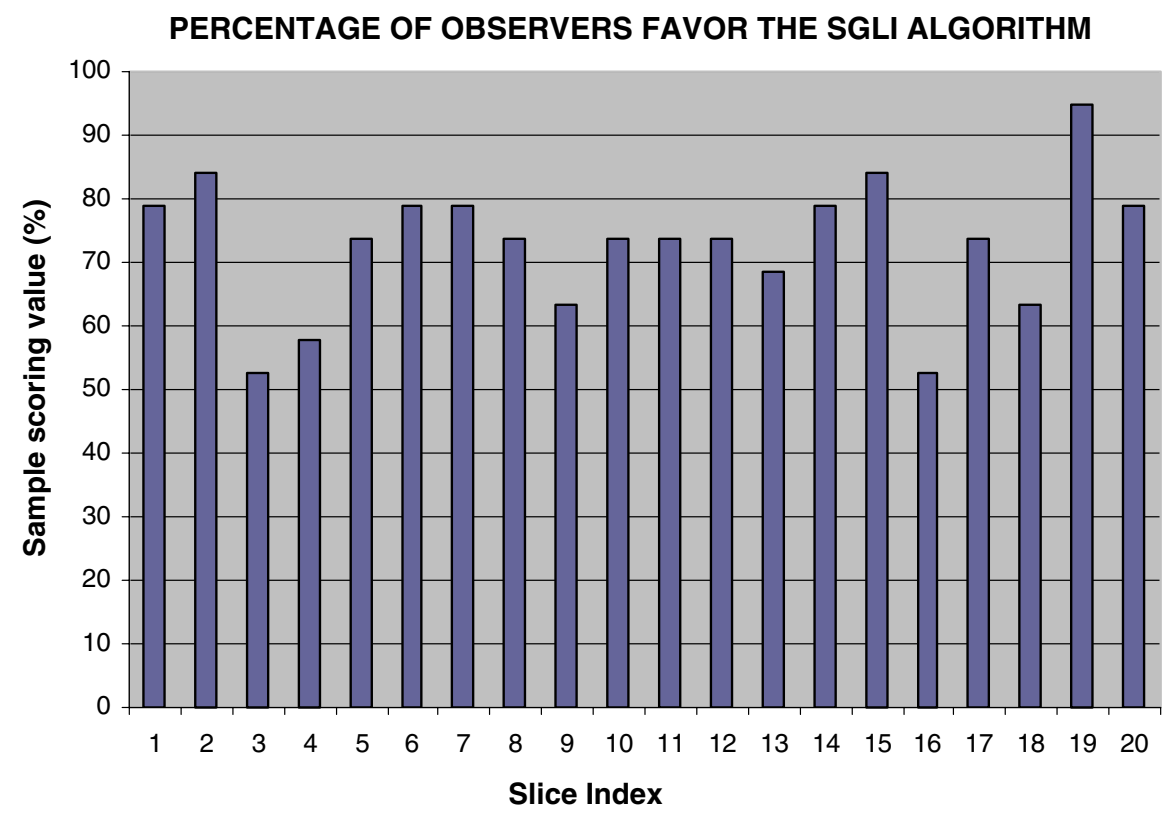


Fig. 8 The observer agreement value for each observer

\section{AGREEMENT WITH THE SENIOR CARDIOLOGIST}

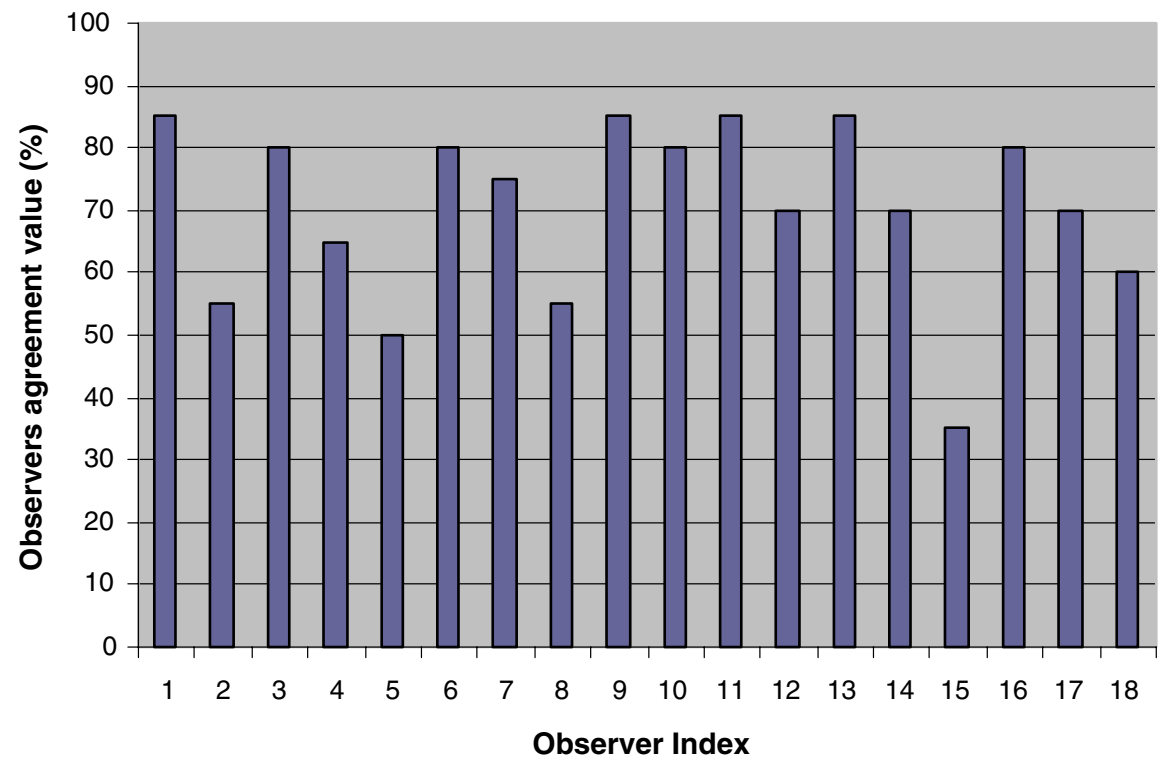

$70.3 \%$ of the scoring samples. The wide range of OAV (from 35.0 to $85.0 \%$ ) indicates that there is large variance in the interobserver agreement, mainly due to the subjectivity of the scoring procedure.

\section{Discussions}

$\mathrm{X}$-ray angiography is one of the standard procedures for the diagnosis of coronary artery diseases. Image enhancement is of great significance to the visual interpretation of vessel abnormalities. However, due to the low contrast property of angiographic images, image enhancement is not a trivial task when strong noise is present. High accuracy in distinguishing interesting objects, e.g., lesions and sidebranches, from background can be extremely difficult in some situations. Therefore, enhancing vessel edges by suppressing background or removing background might as well lose some detailed information, which is very undesirable. Enhancing the whole image content might also decrease the quality of visualization due to the increase of noise level.

One of the widely recognized mechanisms in the eyes of most animals (including humans) for outlining important visual structures is the so-called "lateral inhibition network". While it has great advantage of simplicity, it is not "intelligent" enough to differentiate the noise with the true anatomical structures.
Therefore, enhancement is less optimal when applied to the low contrast angiographic image. The asymmetric sticks, which have better characteristics to fit the patterns of digital image edges, could be used to improve the performance of lateral inhibition models. Instead of removing or suppressing background information to gain better visualization, more effort has been undertaken to distinguish vascular structures from background and lumen by the integration of the stick filtering kernel. The algorithm has low risks of losing detailed information and increasing noise level when enhancing detailed vascular structures on low contrast angiographic images.

Enhancing image details is always desirable for a better visualization quality. However, despite many cardiologists share common opinions about good visualization, there is still no gold standard for defining what the best quality of visualization is. Enhancing detailed information on low contrast images will inevitably increase image noise, which is not always appreciated, especially when the image noise increase significantly. The presence of strong image noise will introduce additional effort in appreciating the interesting information, especially when the cardiologists quickly review the angiographic image sequences for the entire cardiac cycle. On the other hand, reducing noise will potentially increase the chance of losing image details. There is always a trade-off between enhancing details and 
reducing noise. The ultimate goal would be to enhance details to desired quality while keeping the noise at an acceptable level. However, preference of details and tolerance of noise vary among different observers. In addition, it is extremely difficult to define detailed information under certain circumstances. Noise might be accidently treated as information since its presence could create a sense of "details", especially when observers get used to look at the noisy grainy spots on the images. This phenomenon was confirmed by some of the participants in the follow-up discussions after they finished the scoring. It could partly explain the reason why some observers favor the UM algorithm, since they have got used to looking at the images with noisy spots. This phenomenon, together with the subjectivity in step 2 of the scoring procedure, i.e., the judgment of the sharpness of vessel edges, accounts for the big variance in interobserver agreement. On the other hand, despite of all the subjectivities involved, the validation study clearly demonstrated that the participants were in favor of the SGLI enhancement algorithm, mainly due to the reason that the relatively low noise level in the SGLI enhanced images improved the visualization quality and saved the effort for the diagnosis. Although we have not validated the algorithm on cine clips, i.e., running movie, we believe that the relative low noise level and clear image details achieved by the SGLI algorithm could potentially reduce the effort in examining vessel abnormalities and decrease the chance of missing some useful information. Therefore, cardiologists could show more preference on the SGLI algorithm when making quick decisions based on cine clips.

The majority of the computation cost for the proposed algorithm is to calculate the average intensity and variance along each stick for all image points. Current implementation by using $\mathrm{C}++$ language has achieved a speed of 0.09 second per image frame on a Windows PC with $3.0 \mathrm{GHz}$ Core 2 Duo CPU and 2.0 GB RAM. Since each stick in the stick filtering kernel is independent, parallel computing techniques can be applied to further accelerate the algorithm.

One limitation to this study is that all angiographic images used for the validation study were acquired by the same X-ray acquisition system. The quality of original images varies among different acquisition systems and is subject to acquisition conditions, which might influence the enhancement results. However, since the function of SGLI algorithm does not depend on specific noise models and the validation study has already demonstrated its superiority on images with different noise levels, we expect similar results to hold for other image acquisition systems.

\section{Conclusions}

The SGLI algorithm improves the visibility of detailed vascular structures on low contrast coronary angiographic images. The validation study shows that the SGLI algorithm performs significantly better than the UM algorithm.

Acknowledgments We appreciate the participation of all the cardiologists and experts in the validation study. We particularly thank prof. AVG. Bruschke, MD, prof. Y. Ishii, MD, Dr. H. Bezerra, MD, Dr. X. De, B. Stoel, Ph.D for their enlightening discussions.

Open Access This article is distributed under the terms of the Creative Commons Attribution Noncommercial License which permits any noncommercial use, distribution, and reproduction in any medium, provided the original author(s) and source are credited.

\section{References}

1. Reiber JHC, Koning G, Dijkstra J, Wahle A, Goedhart B, Sheehan FH, Sonka M (2000) Angiography and intravascular ultrasound. In: Sonka M, Fitzpatrick JM (eds) Handbook of medical imaging, vol 2. Medical image processing and analysis. SPIE, Washington, pp 711-808

2. van der Zwet PMJ, Reiber JHC (1995) The influence of image enhancement and reconstruction on quantitative coronary arteriography. Int J Card Imaging 11:211-221

3. Aach T, Schiebel U, Spekowius G (1999) Digital image acquisition and processing in medical X-ray imaging. J Electron Imaging 8(1):7-22

4. Kumar MSD, Liyang W, Ram T, Jasjit SS (2007) DSA image enhancement via multi-resolution motion correction for interventional procedures: a robust strategy. Proceedings of the fifth IASTED international conference: biomedical engineering. ACTA Press, Innsbruck

5. WIlson DL, Kaplan EJ (1990) Linear and morphological digital image enhancement of peripheral angiography images. Proceedings of SPIE: medical imaging IV: image processing. Newport Beach

6. Grobstein P (2003) Tricks of the eye, wisdom of the brain. Serendip. Available via webpage. http://serendip.brynmawr. edu/bb/latinhib.html. Accessed 12 Sept 2008 
7. Hartline HK (1956) Inhibition in the eye of Limulus. J Gen Physiol 39:651-673

8. Hartline HK, Knight BW Jr (1974) The processing of visual information in a simple retina. Ann N Y Acad Sci 231:12-18

9. Hartline HK (1969) Visual receptors and retinal interaction. Science 164:270-278

10. Lange D, Hartline HK, Ratliff F (1966) Inhibitory interaction in the retina: techniques of experimental and theoretical analysis. Ann N Y Acad Sci 128:955-971

11. Tu S, Wu Y, Lu X, Huo H, Fang T (2007) Stick-guided lateral inhibition for enhancement of low-contrast image.
Proceedings of SPIE: MIPPR 2007: pattern recognition and computer vision. Wuhan

12. Czerwinski RN, Jones DL, O’Brien WD Jr (1998) Line and boundary detection in speckle images. IEEE Trans Image Process 7:1700-1714

13. Czerwinski RN, Jones DL, O’Brien WD Jr (1999) Detection of lines and boundaries in speckle images - application to medical ultrasound. IEEE Trans Med Imaging 18: 126-136

14. Xiao C, Su Z, Chen Y (2004) A diffusion stick method for speckle suppression in ultrasonic images. Pattern Recognit Lett 25:1867-1877 